\title{
Physical Aspects of Posturography in Virtual Reality
}

\author{
M. KOMAR ${ }^{a}$ AND L. Czerwosz ${ }^{b, *}$ \\ ${ }^{a}$ Faculty of Physics, University of Warsaw \\ ${ }^{b}$ Mossakowski Medical Research Institute, Polish Academy of Sciences \\ Doi: 10.12693/APhysPolA.139.468 \\ *e-mail: lczerwosz@gmail.com
}

\begin{abstract}
Virtual reality is a modern information technology consisting in a computer creation of an image which presents scenes with the participation of objects with the possibility of their movements in virtual space and interaction. The basis of the scene is a mathematical and/or physical model. If the observer can influence the observed scene, then the observation turns into a computer game. A force plate measures the position of the resultant pressure force on the feet (COP) of a person standing on the platform and thus evaluates the involuntary and voluntary swaying of the body, which is constantly losing and regaining its balance. A visual-motor feedback has been used in posturographic research by linking the movements of the COP of a person standing on a force plate with a movement of a virtual marker on the screen against the background of simple geometric figures. Thus, the patient is playing a computer game for which the location of the COP is the information input. The patient's task is to lean forward according to the virtual image of the geometric figure on the screen and maintain the COP marker in the given field for a few seconds. The force of inertia gives the moment of a force about the axis of rotation at the support point of the force plate. Thus, the COP is influenced by inertia forces which results in spurious COP movements at vigorous or abrupt deflections. A piece of Python software has been developed to assess the magnitude of the influence of inertia forces. The model assumes specifying the projection of the centre of gravity (COG) point at the location of the computer mouse instead. The model gives qualitative results which are very close to actual posturographic recordings.
\end{abstract}

topics: virtual reality, force plate, posturography, inertia forces

\section{Introduction}

Virtual reality (VR) is a modern information technology that consists in a computer creation of an image which presents scenes with the participation of objects with the possibility of their movements in virtual space and interaction. The basis of the scene is a mathematical and/or physical model introducing relations between objects - the principles of their movements and mutual influence. If the observer can influence the observed scene by, for example, controlling the position of one of the objects, then the observation of virtual reality turns into a computer game. The essence of the game is a visual-motor feedback. The coupling between patients' movements and the observed virtual scene is explained in this paper.

Virtual reality has found wide application in medicine, including the diagnosis of disturbances of the balance system. VR is also used with great success in the treatment process as an aid to the rehabilitation, e.g., after a stroke, or as a support in the treatment of multiple sclerosis, ataxia and Parkinson's disease.

\section{Virtual reality}

\subsection{Body sways}

In the standing position, the spontaneous or involuntary swaying of the body is observed, which constantly loses and regains its balance.

Generally, three types of body movements can be observed while standing:

- involuntary (IM), including

- automatic,

- random,

- voluntary, intentional.

A good description of voluntary movements related to maintaining the balance is given in [1]. Automatic movements are related to the automation of maintaining balance. The deviation from equilibrium leads to the so-called error signal. First, this signal is the increased muscle tension, which increases with the increasing bending of the body. Secondly, the signal is also the vision, observing 
changes in the direction and position of the usually vertical edges of walls and furniture. This is information that the image seen by the observer or the observer himself changes its/his position. And thirdly, there may be information about the acceleration of the head, spinning - this is a signal from the labyrinth. The signal from the peripheral equilibrium vestibular organs in the inner ear is unfortunately very inaccurate. The generation of an error signal triggers the core muscles or balance strategies such as grip, swing, and leg lunge. The loop of automatic movement has a delay that results in oscillations. Poor feeling of the error signal (low sensitivity of receptors) and delayed muscle response (inability to quickly activate muscle units) could also be causing the loop to run slowly. Lowering the sensitivity and increasing delay increases the amplitude of sways and introduces an additional error. The delayed postural reaction becomes inadequate over time. It can even cause a deterioration in body position and even a complete loss of balance.

\subsection{Posturography}

A force plate (Fig. 1) measures the position of the resultant pressure force on the feet (COP) of a person standing on a platform and thus evaluates the involuntary swaying (and voluntary movements) of the body, which is constantly losing and regaining its balance. The evaluation of this functionality is carried out by parameterizing the trajectory of the COP movements. Posturography allows for an objective assessment of the balance system.

The instantaneous position of the COP point can be calculated using [2]:

$$
x(t)=\frac{d}{2} \frac{\left(-p_{1}+p_{2}+p_{3}-p_{4}\right)}{\left(p_{1}+p_{2}+p_{3}+p_{4}\right)}
$$

and

$$
y(t)=\frac{d}{2} \frac{\left(p_{1}+p_{2}-p_{3}-p_{4}\right)}{\left(p_{1}+p_{2}+p_{3}+p_{4}\right)},
$$

where $p_{i}$ are the time-dependent forces measured on four supports with precision tensometers, and $d$ is the side length of the force plate. The trajectory of the COP motion shown in the $X Y$ system is

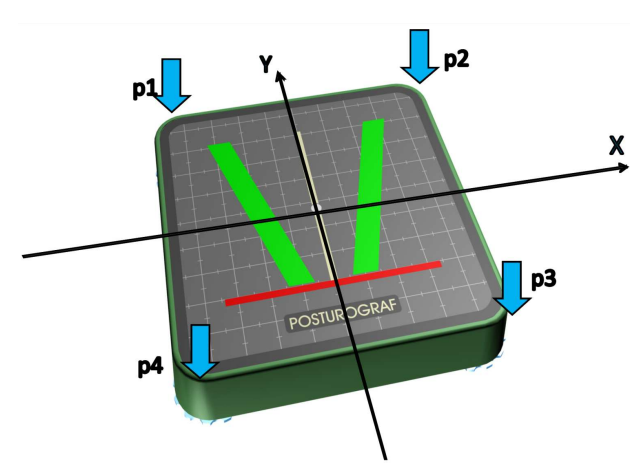

Fig. 1. Force plate [2]. The patient stands on the platform. The forces $p_{1}, p_{2}, p_{3}, p_{4}$ are acting against four supports. a statokinesiogram [3]. Presenting the history of COP movements - the $x$ and $y$ positions as functions of time - is a stabilogram [3].

\subsection{Inertia forces in standing position}

In the standing posture, the human body is in a position of unstable balance. This position is maintained thanks to the existence of a balance system that analyzes the position, movement and acceleration of the body and activates or releases the corresponding core muscles to restore the upright position. Inverted pendulum models play an important role in modelling the vertical position [4]. The force of inertia directed horizontally gives the moment of a force about the axis of rotation at the support point of a force plate so that the moment of inertia is added to the moment of gravity, and this affects the resultant weight and its point of attachment COP (measured by the force plate).

\subsection{Visual-motor feedback}

The visual-motor feedback has been used in posturographic research by linking the movements of the COP of a person standing on the force plate with a movement of a virtual marker on the screen against the background of simple geometric figures.

The location of the COP point is influenced by:

- the position of the vertical projection on the horizontal plane of the centre of gravity $(\mathrm{COG})$ or the centre of mass (COM) of the body,

- the inertia forces generated by body sways, i.e., inclinations from the vertical and by reupright standing.

The body performs accelerated movements with constantly changing the acceleration direction and value, which results from the activation or relaxation of the postural muscles and the position of the body about the force of gravity. A patient standing on the force plate can actively participate in the virtual reality scene when the following loop is being performed [5]:

- the patient observes the scene on the screen, it could be a simple geometric picture, e.g., $2 \mathrm{~cm}$ ahead of the central position and the marker (black rectangle, but it can be any object in the scene visualization),

- the patient is leaning out adequately to the scene (or inadequately if he cannot cope with the difficulties of the exercise), let the patient move by dragging the marker $2 \mathrm{~cm}$ forward,

- the platform measures a new position of the COP,

- the object on the screen (marker showing the position of the COP) moves - the scene changes,

- end of the loop. 
The above loop is repeated many times a second. Each time the position of the object/objects is/are fully computed, then the scene is rendered. The person standing on the force plate uses his muscles, activates and releases the muscle units in the core muscles to perform the intended movements to keep the marker in the area marked by the circle or square.

Observation of the marker representing COP significantly increases the possibility of feeling slight body tilts. A tilt of a few milimeters is usually imperceptible for most people with a disturbed balance system, and even for healthy people. Very sensitive measurement of deviations from the central position with an accuracy of about $0.1 \mathrm{~mm}$, which is performed by the apparatus, allows the conscious activation of the body position correction mechanism based on the visible shift of the marker on the screen. Comparison of the parameters of the COP trajectory of spontaneous sways recorded with eyes open and the COP trajectory in the feedback gives information about the state of deep sensation receptors, which should effectively inform about excursions. The assessment may be quantitative if a simple scoring system is introduced, e.g., the proportion of the time the COP marker remains in the marked field to the time of the exercise.

\section{Method}

\subsection{Posturography}

The experiment consisted in the posturographic measurement of the person on the force plate and performing alternately forward-leaning and straightening to a standing upright position. The displacements of the COP point had an amplitude of $2-5 \mathrm{~cm}$. The COP trajectory was recorded in a computer system for $32 \mathrm{~s}$ with sampling frequency of $32 \mathrm{~Hz}$, and resolution of 12 bits. The analogue signal was previously filtered with a low-pass filter with a cut-off frequency of $15 \mathrm{~Hz}$. The results were presented in the form of a stabilogram taking into account only the $y(t)$ component of the COP movement, as the deflections were only forward. The exercise had to be done once quickly and once slowly. Faster movements caused the generation of larger inertia forces, which significantly affected the position of the COP point, moving it towards or opposite to the return of body movement. In posturographic measurements, only the resulting location of the COP point is known, the position of the COG cannot be measured.

\subsection{Computer mouse model}

A piece of Python software has been developed to assess the magnitude of the influence of inertia forces. It allows visualizing the location of the centre of gravity, speed and acceleration by digital calculation of the first and the second derivative. The model assumes specifying the COG point at the location of the computer mouse instead of the projection of the centre of gravity. The position of the mouse is determined by the human hand, just as the position of the centre of gravity is determined by the position of the entire body. The COP is partially defined by the position of the COG and partially by the second derivative with respect to time. Thus, the COP point was modelled as the following combination:

$$
\operatorname{COP}(x, y)=\mathrm{COG}+W \frac{\mathrm{d}^{2} \mathrm{COG}(x, y)}{\mathrm{d} t^{2}},
$$

where $x$ and $y$ determine the position of the COP and COG points on the plane and $W$ determines the influence of the inertia forces. A mild one-sided smoothing was used to calculate the derivatives. Calculations were made in real-time so that it was possible to present on-line the COP point simultaneously with the COG, which was determined by the mouse position.

\section{Results and discussion}

The qualitative results of the model's operation are shown in Fig. 2.

In a doctoral dissertation [6], as a control group, young healthy men aged $21-23$ were analyzed. The task was to keep the marker inside the virtual square.

Young men performed the exercises very vigorously, which completely spoiled their results. The score calculated as a percentage of the time the marker was inside the square was approximately $30 \%$, while patients with imbalances obtained 60 $70 \%$, and the elderly healthy people - even $90 \%$, mainly due to visual impairment.

Our experiment explains the very bad results of the group of student youth that was supposed to be the norm group. The cause of the poor stabilization of the COP point can be attributed to the inertia forces. The more the person standing on the force plate tried to stop the marker movement, the faster the marker moved and escaped from the target square. Our model explicitly explains the influence of inertia forces on medical tests with feedback and posturographic computer games [7]. A qualitative explanation can now be provided without difficulty. There are four phases of swaying posture:

(I) tilting the body from the central position to the front,

(II) stopping forward-leaning,

(III) starting the return to the vertical upright position,

(IV) stopping straightening.

Ad. (I) The phase of tilting the body from the central position to the front: the COG moves forward - initially, it is a positively accelerated movement (the body is just starting) — the inertia force is, therefore, opposite to the direction of movement. 


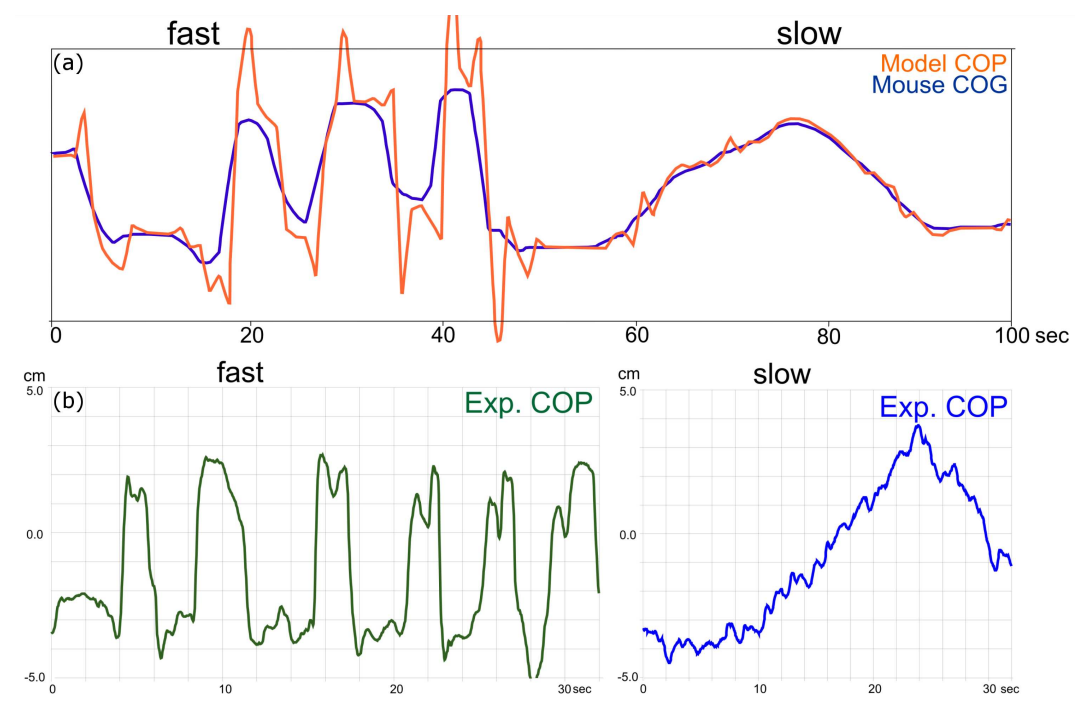

Fig. 2. (a) Computer mouse model chart: The modelled COP instantaneous position (orange) and the COG position - a real mouse position in the experiment (blue). As you accelerate the mouse, the COP point follows the COG point. During deceleration, the COP is ahead of the COG. (b) The actual experimental posturographic stabilograms. The curves are very similar to modelled graphs (orange). The graph on the left (green) shows the experimental COP while fast movements-tilting are being performed on a force plate with a quick return to the upright posture. Slow movements are shown on the right (blue).

The moment of this force about the support points works in such a way that the pressure on the back of the platform is greater than on the front. There is, therefore, a recession of the COP compared to the vertical projection of the COG movement. As a result, the COP moves towards the front slower than it would result from the static-geometric projection of the COG on the horizontal plane. The COP is closer to the central point than the vertical projection of the COG. The viewer of the screen gets the feeling that he is leaning too slowly and tilting faster, which is inappropriate.

Ad. (II) Stopping the tilting forward, the movement is still taking place, but it is the movement negatively accelerated due to the activation of the posture muscles, for example in the ankle joint. The COG vertical projection point continues to move forward. The inertia force is now in line with the direction of movement - additional pressure is placed on the front of the feet, the origin of the pressure of inertia. As a result, the COP moves faster towards the front than it would result from the staticgeometric calculus. The forward deflection of the COP from the centre point is larger as compared to the vertical projection COG. The observer of the screen has the impression that tilting takes too long, the person brakes even more. Due to inadequate visual information, the return to the vertical position begins too early and too abruptly.

Ad. (III) Starting to return to the vertical position: the COG leaned forward starts to reverse - there is a positively accelerated movement backwards. The inertia force is opposite to the direction of movement (backwards), so it is directed forwards - this results in pressure to the front of the feet.
In the same way as in phase (ii), the forward deflection of the COP from the central point is still larger as compared to the vertical projection of the COG, although the COG is just being reversed because the body is returning to its vertical position. As a result, the viewer of the screen has the impression that straightening is too low and therefore accelerates, which further delays the COP in pursuit of the COG.

Ad. (IV) Stopping the straightening, the body approaches the vertical position, the COG approaches the central position - it is a negatively accelerated backward movement. The inertia force is consistent with the direction of movement, i.e., it is directed backwards. Due to the inertial force, there is additional pressure on the back of the plate as in phase (I), which makes the COP point closer to the centre point as compared to the COG vertical projection. The abrupt braking of the straightening phase causes the COP to move backwards beyond the central position, which is a visual signal for unnecessary forward movement.

Feedback exercises are mainly done for training. Patients with dysfunctions of the motor system or the equilibrium system learn to move in virtual space. The movement strategy is to perform gentle, moderately fast displacements of the COG point so that the body does not accelerate too much. Then the COP point does not deviate significantly from the COG. The condition for such a movement is to control random, involuntary movements and adjust the force to the needs. This is the main purpose of the training. Paradoxically, therefore, the forces of inertia contribute to a more favourable way of performing the exercises for the neuromuscular system. 


\section{References}

[1] L.I. Oddsson, Acta Physiol. Scand. Suppl. 595, 1 (1990), PMID: 2080712.

[2] L. Czerwosz, E. Szczepek, B. Sokołowska, J. Jurkiewicz, Z. Czernicki, Recognition of Posture and Gait Disturbances in Patients with Normal Pressure Hydrocephalus Using a Posturography and Computer Dynography Systems, in: Hydrocephalus, Eds. S. Pant, I. Cherian, Intech, 2012.

[3] M. Duarte, S.M.S.F. Freitas, Rev. Bras. Fisioter. 14, 183 (2010).

[4] T.C. Winter, J.W. Harvey, O.L. Franke, W.M. Alley, Circular 1139, 79 (1998).
[5] L. Czerwosz, J. Błaszczyk, M. Mraz, M. Curzytek, in: 2009 Virtual Rehabilitation Int. Conf., Haifa (Israel), 2009, p. 214.

[6] R. Leszcz, Ph.D. Thesis, the Warsaw Medical University, 2020 (Unpublished).

[7] I. Strumecka, D. Hawryluk, "Implementation of the Game System for the Rehabilitation of People with Labyrinthitis", Dissertation for the degree of an engineer, the University of Wroclaw, the Faculty of Mathematics and Computer Science, the Institute of Computer Science (in Polish). 